\title{
Osteogenic differentiation of human periodontal ligament stem cells expressing lentiviral NEL-like protein 1
}

\author{
CAI-YUN CHEN ${ }^{1 *}$, YA-JING LIU ${ }^{2 *}$, SHENG-GEN SHI $^{3}$, FA-MING CHEN $^{4}, \mathrm{CHUAN} \mathrm{CAI}^{5}$, BING LI $^{2}$, \\ JING WANG ${ }^{5}$, LIANG SHI ${ }^{3}$, YAN LI ${ }^{3}$, ZHONG-YU LIU ${ }^{6}$ and ZHONG-YING NIU ${ }^{3}$ \\ ${ }^{1}$ Chinese PLA Postgraduate Medical School, Beijing; ${ }^{2}$ Institute of Stomatology, Chinese PLA General Hospital, Beijing; \\ ${ }^{3}$ Center of Stomatology, The 306th Hospital of PLA, Beijing; ${ }^{4}$ Center Laboratory for Tissue Engineering, School \\ of Stomatology, ${ }^{5}$ Department of Orthodontics, School of Stomatology, The Fourth Military Medical University, \\ Xi'an, Shanxi; ${ }^{6}$ Department of Gynecology, Chinese PLA General Hospital, Beijing, P.R. China
}

Received April 7, 2012; Accepted June 15, 2012

DOI: $10.3892 /$ ijmm.2012.1053

\begin{abstract}
NEL-like protein 1 (NELL1) is a newly identified secreted protein involved in craniosynostosis and has been found to promote osteogenic differentiation of mesenchymal stem cells. The objective of this study was to investigate the effect of NELL1 on osteogenic differentiation of human periodontal ligament stem cells (hPDLSCs) and the potential underlying mechanism. hPDLSCs underwent lentivirus-mediated NELL1 transfection (Lenti-NELL1) and markers of osteogenesis were assessed [alkaline phosphate (ALP), osteocalcin (OCN) and calcium deposition] to evaluate the effect of NELL1 on the differentiation of these cells. Quantitative polymerase chain reaction (qPCR) was employed to measure the mRNA expression of Msx 2 and Runx2, and Lenti-enhanced green fluorescent protein (EGFP) served as a control. Western blot analysis and qPCR analyses confirmed that Lenti-NELL1-transfected hPDLSCs could express NELL1. Compared with the LentiEGFP group, ALP, OCN, calcium deposition and Msx 2 mRNA expression were markedly increased $(\mathrm{P}<0.01)$, but there was no significant difference in Runx 2 mRNA expression between the two groups $(\mathrm{P}>0.01)$. hPDLSCs can be transfected by Lenti-NELL1 and can stably express NELL1. NELL1 is able to promote the osteogenic differentiation of hPDLSCs, which may be related to the downregulation of Msx 2 expression. Lenti-NELL1 transfection can be used during in vitro gene therapy for periodontal regeneration.
\end{abstract}

\section{Introduction}

Periodontal diseases result in damage to periodontal tissues, including the periodontal ligament (PDL), cementum, and

Correspondence to: Professor Zhong-Ying Niu, Center of Stomatology, the 306th Hospital of PLA, Beijing 100101, P.R. China E-mail: niuzhongyinpro@126.com

${ }^{*}$ Contributed equally

Key words: lentivirus, NEL-like protein 1, human periodontal ligament stem cell, osteogenic differentiation alveolar bone, and are the main cause of tooth loss in adults. Periodontal diseases are a health burden worldwide (1). Reconstruction of healthy PDLs is a major goal in the treatment of periodontal diseases. Human periodontal ligament stem cells (hPDLSCs) have the potential to form PDL, cementum, and alveolar bone and are ideal seed cells for periodontal tissue engineering.

Bone morphogenetic proteins (BMPs) are a group of classic bone growth factors that can exert osteogenic effects on a broad spectrum of cell types. At the same time, BMPs have been found to be involved in the development of numerous tissues and organs $(2,3)$, and this nonspecificity of BMPs usually results in unexpected side effects $(4,5)$. Thus, it is imperative to identify growth factors with fewer side effects and favorable specificity as an alternative to BMPs. NEL-like protein 1 (NELL1) is a secreted protein related to human craniosynostosis (CS) (6) that can specifically act on osteochondral lineage. NELL1 has been demonstrated robust induction of bone in multiple animal models $i X n$ vivo (7-9). Different from BMP-2, NELL1 cannot independently induce ectopic osteogenesis in muscle (10). NELL1's unique role as a novel osteo-inductive factor makes it an attractive and promising future in clinical practice. However, few studies have reported the osteogenic effect of NELL on hPDLSCs.

Studies have confirmed that recombinant NELL1 protein can promote the osteogenesis of mesenchymal stem cells (MSCs) and subsequent bone formation (11). However, proteins may spread or be inactivated and the dose of recombinant proteins is usually large in clinical practice $(12,13)$, which then results in unexpected side effects and increases the cost. Regional gene therapy has been regarded as an effective strategy to resolve this limitation (14). Adenovirus-mediated NELL1 gene therapy (AdNELL1) has been indicated to induce bone regeneration in animal models $(7,15)$. Bone repair is a long-term process and adenoviruses cannot support lasting expression of target genes (16). However, lentiviral vectors can effectively and stably express target genes by integrating their DNA into the host genome. Recent studies have also confirmed that lentiviruses expressing BMP-2 are superior to adenoviruses expressing BMP-2 in gene therapy for bone regeneration (17). This suggests that lentivirus expressing 
NELL1 (Lenti-NELL1) may be a favorable candidate for bone regeneration.

In the present study, Lenti-NELL1 was constructed and the feasibility of using virus to infect hPDLSCs was investigated. In addition, the effect of NELL1 on the osteogenic differentiation of hPDLSCs following lentivirus infection and the potential underlying mechanisms were studied. Our results showed that Lenti-NELL1 infected hPDLSCs could effectively express bioactive NELL1 over a long period of time and that NELL1 could further promote the osteogenic differentiation of hPDLSCs in runt-related transcription factor 2 (Runx2) independent manner. In summary, our findings demonstrated that Lenti-NELL1 transfection of hPDLSCs may be a promising strategy for bone and periodontal tissue engineering.

\section{Materials and methods}

Isolation of human PDLSCs. This study was approved by the Ethics Committee of the General Hospital of People's Liberation Army, and informed consent was obtained before beginning the study. A total of 40 impacted, caries-free molars were extracted from 28 patients. The PDL was gently collected and digested in $3 \mathrm{mg} / \mathrm{ml}$ type I collagen (Sigma-Aldrich, St. Louis, MO, USA) and $4 \mathrm{mg} / \mathrm{ml}$ dispase (Roche Diagnostics $\mathrm{GmbH}$, Mannheim, Germany) for $1 \mathrm{~h}$ at $37^{\circ} \mathrm{C}$ (18). PDL samples from different patients were pooled, and a single cell suspension was prepared and filtered through a $200-\mu \mathrm{m}$ pore size filter. Cells were maintained in basic medium $(\alpha$-MEM containing 10\% FBS; Gibco-BRL, Grand Island, NY, USA) at $37^{\circ} \mathrm{C}$ in $5 \% \mathrm{CO}_{2}$. The density of cells in the logarithmic growth phase was adjusted to $10-15$ cells $/ \mathrm{ml}$, cells were then seeded into a 96-well plate (100 $\mu 1 /$ well) followed by incubation for $12 \mathrm{~h}$. The medium was added at a final volume of $200 \mu \mathrm{l}$ in each well. When cell confluence covered 30-50\% of the bottom of the well, the cells were digested in $0.25 \%$ trypsin (SigmaAldrich) and passaging was performed to acquire single clones and subclones of stable PDLSCs.

Characterization of human PDLSCs. Immunofluorescence staining with STRO- 1 was performed to identify PDLSCs. The purified stem cells were seeded into plates followed by incubation for $24 \mathrm{~h}$. After washing in PBS, cells were fixed in $2 \%$ p-formaldehyde for $15 \mathrm{~min}$. These cells were then incubated with mouse anti-human STRO-1 (Millipore, Billerica, MA, USA) for $12 \mathrm{~h}$ and goat anti-mouse IgM (SouthernBiotech, Birmingham, AL, USA) in the dark for $40 \mathrm{~min}$. To detect the multipotency, hPDLSCs were incubated in osteogenic and adipogenic media (Gibco-BRL, Grand Island, NY, USA) for 21 days, alizarin red staining and oil red $\mathrm{O}$ staining were performed to detect the osteoblast- and adipocyte-like cells, respectively. hPDLSCs maintained in basic medium served as controls.

Construction of the plasmid expressing NELL1 (plentiNELL1-IRES-EGFP). The ViraPower Lentiviral Expression System (Invitrogen, Carlsbad, CA, USA) was employed to synthesize human NELL1 (NM006157.3) with the whole genome synthetic method. A single strand oligonucleotide was first synthesized and restriction sites (BamHI and NheI) were included at both ends. Standard overlap PCR was performed to ligate the synthesized oligos into a complete sequence, which was then introduced into pMD-18T vectors (Takara Bio, Inc., Shiga, Japan). The pMD-18T vectors were used to transform competent DH5 $\alpha$ cells (Invitrogen, Carlsbad, CA, USA), and overlap PCR was performed to repair the mutated sites. Restriction enzymes (BamHI and NheI; Fermentas, Inc., Glen Burnie, MD, USA) were used to treat the sequence, and the products were ligated into the target vector plenti-MCS-IRESEGFP, which was then transformed into competent Stb13 cells (were from Invitrogen). Sequencing was performed to determine the inserted sequence and whether plenti-NELL1IRES-EGFP could express NELL1.

Viral packing and detection of viral titer. The plentiNELL1-IRES-EGFP vectors were mixed with Packing Mix, Lipofectamine 2000, and Opti-MEM (were from Invitrogen) according to the manufacturer's instructions. This mixture was then added to $293 \mathrm{~T}$ cells followed by incubation at $37^{\circ} \mathrm{C}$ for $6 \mathrm{~h}$. The medium was refreshed with DMEM containing $10 \%$ FBS followed by incubation for $48 \mathrm{~h}$. The supernatant was collected and centrifuged at $50,000 \times \mathrm{g}$ for $2 \mathrm{~h}$ at $4^{\circ} \mathrm{C}$. The virus particles were resuspended and stored at $-80^{\circ} \mathrm{C}$. The virus suspension was serially diluted, which was then added to HEK293 cells. Fluorescence microscopy was performed to determine the required titer of the virus.

Cell transfection. hPDLSCs with favorable growth were seeded into plates. When cell confluence reached $>80 \%$, the cells were incubated in DMEM containing 5\% FBS followed by addition of virus suspension at multiplicities of infection (MOIs) of 0,10, 50, 100, 150 and 200. A total of $12 \mathrm{~h}$ later, the cells were incubated with basal medium for $60 \mathrm{~h}$. Then, cells that were positive for green fluorescence were counted and the cell status was also observed.

Western blot analysis. Cells were harvested 14 days after lentivirus transfection. The cell lysate was subjected to SDS-PAGE for protein separation. The proteins were then transferred onto a PVDF membrane (Millipore), which was subsequently incubated with $5 \%$ non-fat milk for $1 \mathrm{~h}$. The membrane was then treated with mouse anti-human NELL1 (Sigma-Aldrich) at $4^{\circ} \mathrm{C}$ overnight and then with a goat anti-mouse secondary antibody (Santa Cruz Biotechnology, Inc., Santa Cruz, CA, USA) for $1 \mathrm{~h}$. GAPDH served as an internal reference (Shanghai KangCheng Bio-tech, Shanghai, China). Visualization was performed with ECL (Thermo Fisher Scientific, Rockford, IL, USA). A FluorChem HD2 gel image system (ProteinSimple, Santa Clara, CA, USA) was employed to determine the expression of the target protein.

Proliferation assay. Cells were seeded into a 96-well plate $\left(2 \times 10^{4}\right.$ cells/well), and an MTS kit (Promega Corporation, Madison, WI, USA) was employed to detect cell proliferation (OD at $490 \mathrm{~nm}$ ) (19).

Alkaline phosphatase activity assay. A colorimetric method was used to measure alkaline phosphate (ALP) activity according to the manufacturer's instructions (Nanjing Biotechnology, Nanjing, China) (19). OD was measured at $490 \mathrm{~nm}$. 
Table I. Primer sequences.

\begin{tabular}{lll}
\hline Human genes name & Forward primer sequence $\left(5^{\prime} \rightarrow 3^{\prime}\right)$ & Reverse primer sequence $\left(5^{\prime} \rightarrow 3^{\prime}\right)$ \\
\hline NELL1 & GCTTTGGGATGGACCCTGAC & GAAATAAAAATGCTTTGCTGGC \\
Runx2 & CTCTACTATGGCACTTCGTCAGG & GCTTCCATCAGCGTCAACAC \\
Msx2 & AGATGGAGCGGCGTGGAT & TGGAGGGCAGCATAGGTTT \\
GAPDH & GTCTCCTCTGACTTCAACAGCG & ACCACCCTGTTGCTGTAGCC \\
\hline
\end{tabular}

Alizarin red staining. Samples were fixed in $10 \%$ formalin for $15 \mathrm{~min}$ and then in $1 \%$ alizarin red for $2 \mathrm{~min}$. Following washing in water, alizarin red was quantitated. In brief, samples were washed with $10 \%$ cetylpyridinium chloride (CPC) to remove the alizarin red and spectrophotometry was performed to measure the OD at $490 \mathrm{~nm}$ (20). Detection was done in triplicate and each experiment was performed twice.

Real-time PCR analysis. Total-RNA was extracted with TRIzol reagent (Invitrogen). BioPhotometer Plus (Eppendorf, Hamburg, Germany) was employed to measure the concentration and purity of the RNA. A RevertAid First Strand cDNA Synthesis kit (Fermentas, Inc.) was used to synthesize first strand cDNA, and PCR was performed with SYBR-Green Real-Time PCR Master Mix (Applied Biosystems, Foster City, CA, USA). The mRNA expression of NELL1, Runx2, $M s \times 2$ and GAPDH was detected using an ABI PRISM 7500 Real-time PCR system (Applied Biosystems, Foster City, CA, USA). GAPDH served as an internal reference, and the mRNA expression of the target genes was normalized to that of GAPDH (14). Specific human genes and primer sequences are listed in Table I.

ELISA for osteocalcin. At 7, 14 and 21 days after transfection, ELISA (Invitrogen) was performed to measure osteocalcin (OCN) in the supernatant at $490 \mathrm{~nm}$, and the OD value was recorded.

Statistical analysis. Data are presented as the mean with standard deviation. The comparisons between the 2 groups were tested with the independent two-sample t-test. P-values $<0.05$ were considered statistically significant. Statistical analyses were performed using SPSS v15.0 statistics software (SPSS, Chicago, IL, USA).

\section{Results}

Characterization of human PDLSCs. After digestion with collagenase, hPDLSCs were successfully collected and $>90 \%$ of the cells were observed to be positive for STRO-1 (Fig. 1A and B). To identify the multipotency of the isolated cells, the hPDLSCs were cultured in osteogenic and adipogenic media for 21 days, and the differentiation into these lineages was confirmed by alizarin red and oil red $\mathrm{O}$ staining, respectively (Fig. 1C and D).

Gene transduction and the effects on cell proliferation. In order to establish the optimal MOI for high lentivirus gene

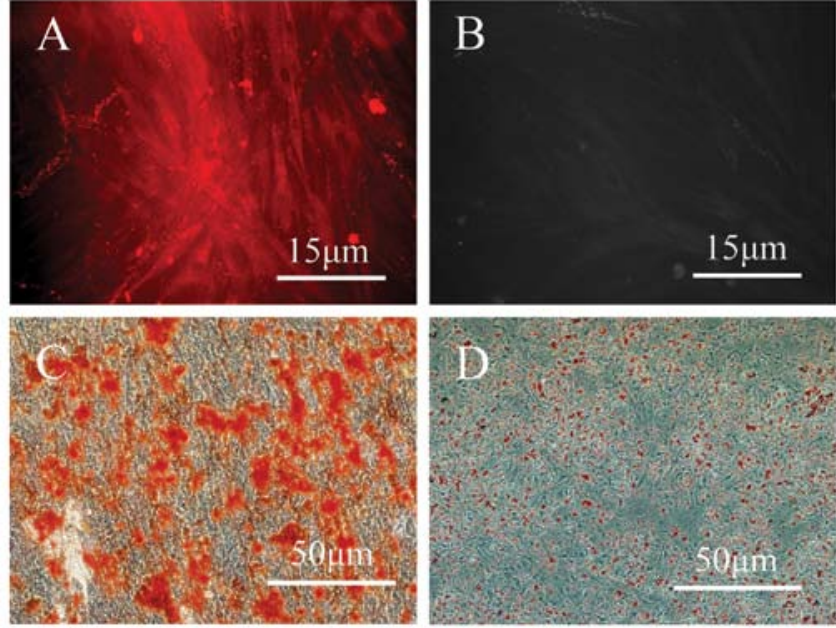

Figure 1. Characterization of hPDLSCs. (A and B) Cells identified as stem cells by immunohistochemistry for STRO-1. (C and D) Differentiation capacity of isolated hPDLSCs. Differentiation was induced by incubating hPDLSCs in differentiation media for 3 weeks. Following fixation, the cells were stained by alizarin red or oil red $\mathrm{O}$, as appropriate.

transfer efficiency, a series of MOIs were assessed in this study. A MOI of $100 \mathrm{pfu} / \mathrm{cell}$ achieved high transfer efficiency above $90 \% 72 \mathrm{~h}$ after lenti-EGFP transduction of hPDLSCs were positive for green fluorescence (Fig. 2C and D). While in the Lenti-NELL1 group, more than $90 \%$ of cells were positive for green fluorescence only when the MOI was $150 \mathrm{pfu} / \mathrm{cell}$ (Fig. 2E and F). In addition, the morphology of the cells in the Lenti-NELL1 group was similar to that in the Lenti-EGFP group (Fig. 2A and B). Quantitative polymerase chain reaction (qPCR) showed that the mRNA expression of NELL1 in the Lenti-NELL1 group at 7 and 14 days after transfection was increased 7.02- and 9.23-fold $(\mathrm{P}<0.001)$, respectively, when compared with that in the Lenti-EGFP group at Day 0 (Fig. 2G). Western blotting assays also demonstrated that cells in the Lenti-NELL1 group expressed NELL1 (Fig. 2H).

The proliferation and differentiation of stem cells are 2 opposing processes. Cells with high differentiation usually have low proliferation and those with high proliferation often present with low differentiation (21). Thus, theoretically, NELL1 may not only promote the differentiation of hPDLSCs but also inhibit their proliferation. A previous study showed that AdNELL1-transfected goat MSCs inhibited proliferation (7). As expected, 1 and 2 days after transfection, the proliferation of cells in the Lenti-NELL1 group was comparable to that in the Lenti-EGFP group. However, 3 days after transfection, 

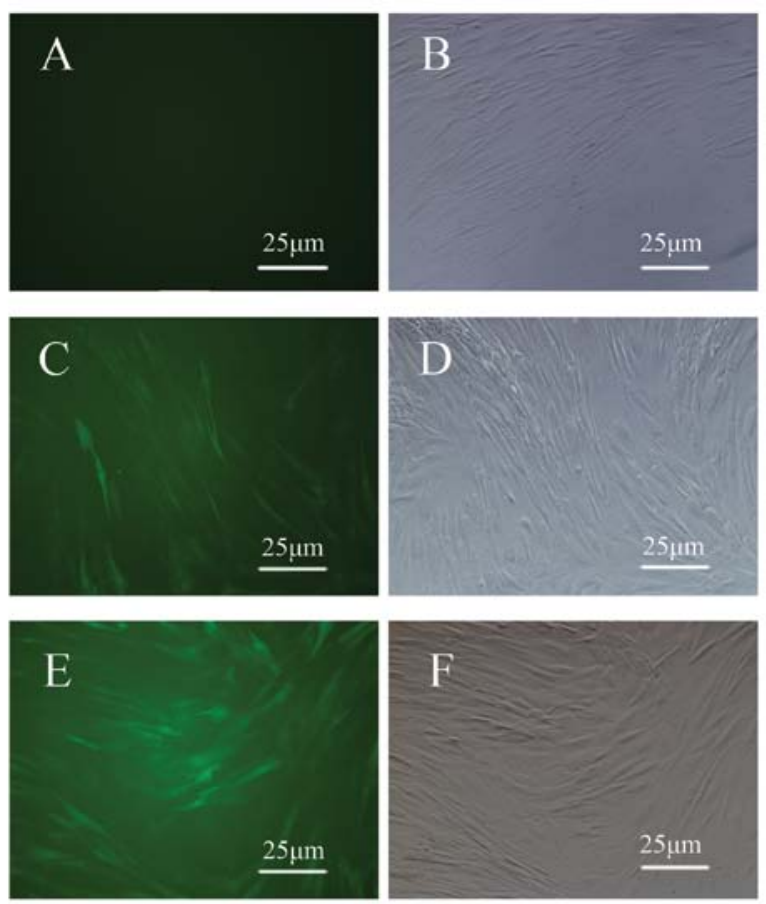

G

$\mathrm{H}$

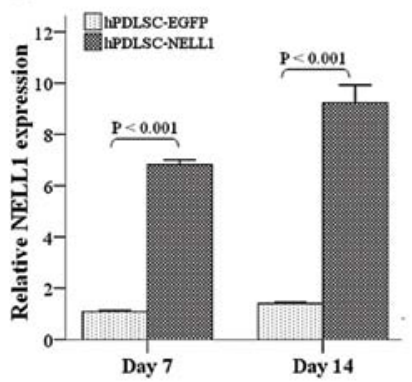

hPDLSC-EGFP hPDLSC-NELL1

NELL1

GAPDH

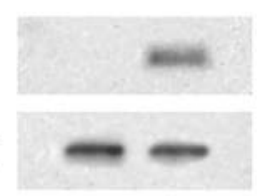

Figure 2. Gene transduction. (A and B) Absence of GFP expression in hPDLSCs without transduction of any lentivirus. (C and D) A MOI of $100 \mathrm{pfu} /$ cell achieved a high transfer efficiency (above $90 \%$ ) at $72 \mathrm{~h}$ after Lenti-EGFP transduction of hPDLSCs, as indicated by positive GFP expression. (E and F) A MOI of $150 \mathrm{pfu} /$ cell achieved a high transfer efficiency (above 90\%) at $72 \mathrm{~h}$ after Lenti-NELL1 transduction of hPDLSCs, as indicated by positive GFP expression. Original magnification, $\mathrm{x} 200$. (G) qPCR analysis of NELL1 in hPDLSCs transduced with Lenti-EGFP or Lenti-NELL1. (H) Western blot analysis probed with antibodies against NELL1 and GAPDH for confirmation of NELL1 protein expression 14 days after gene transduction.

the proliferation of hPDLSCs in the Lenti-NELL1 group was markedly lower than that in the control group $(\mathrm{P}<0.01)$ (Fig. 3).

Osteogenic differentiation of hPDLSCs in vitro after gene transduction. Under appropriate conditions, PDLSCs may differentiate into multiple cells, including osteoblasts and adipocytes $(18,22)$. In the present study, cells transfected with Lenti-EGFP served as controls and the osteogenic effect of NELL1 was determined. After transfection, these cells received osteogenic induction. ALP is a marker of early osteogenesis. The results showed that, as compared to the control group, cells in the Lenti-NELL1 group had comparable ALP activity at Day 0 but showed significantly increased ALP activity 6 days after induction $(\mathrm{P}<0.001)$ (Fig. 4A). For the osteogensis late indicator of OCN, their production was similar to that above. At 7 and 14 days after

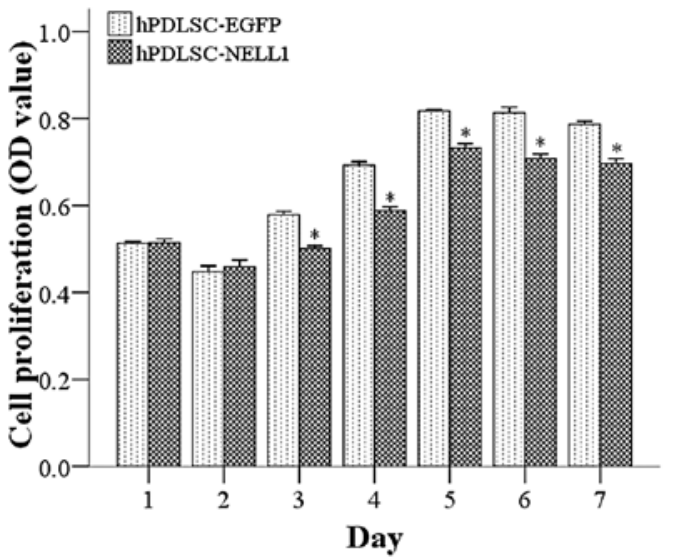

Figure 3. Effects of Lenti-EGFP and Lenti-NELL1 infection on hPDLSC proliferation as analyzed by MTS assay. Comparison of cellular absorbance between the Lenti-NELL1 and Lenti-EGFP groups. ${ }^{*} \mathrm{P}<0.001$.

induction, OCN levels in the Lenti-NELL1 group were markedly higher than in the control group $(\mathrm{P}<0.001)$ (Fig. 4B). In addition, alizarin red staining showed that more calcium was found in the Lenti-NELL1 group than in the Lenti-EGFP group (Fig. 4C and D). Quantitative analysis showed that calcium mineralization was more evident in the Lenti-NELL1 group than in the control group $(\mathrm{P}<0.001)$ (Fig. 4E). On the other hand, NELL1 is a secreted protein. To further validate whether NELL1 in Lenti-NELL1-transfected hPDLSCs was biologically active, the supernatant was mixed with normal osteogenic media made of the conditional medium at a ratio of 1:1 for osteogenic cultured 21 days. The results showed more calcium deposition in the Lenti-NELL1 than in the control group $(\mathrm{P}<0.001)$ (Fig. 4F-H). The above findings indicated that Lenti-NELL1-transfected hPDLSCs were able to successfully express bone-inducing active NELL1 protein that could vigorously facilitate the osteogenic differentiation of hPDLSCs at early and late stages.

To explore the probable mechanism underlying the promotion of PDLSCs osteogenic differentiation by NELL1, two primary transcription factors (Runx2 and Msx2) involved in osteogenic differentiation were investigated. Studies have demonstrated that NELL1 is a downstream factor of Runx 2 and is directly regulated by Runx2 (23). As expected, although Runx 2 mRNA expression in the Lenti-NELL1 group at 7 and 14 days after transfection was increased 11.17 and 10.05-fold when compared with that in the control group at Day 0, no distinct difference was noted between the two groups ( $\mathrm{P}>0.01)$ (Fig. 4I). Msx2 is an important regulator in osteogenesis, especially in the Runx2 independent signaling pathway. Interestingly, like NELL1, Msx2 is also highly related to CS and plays an equally important role in craniofacial development (24). When compared with the control group at Day 0, Msx2 mRNA as compared with the control group at Day 0, the Lenti-NELL1 group at Day 7 was increased by 4.6-fold higher than that in the control group (2.61-fold). ( $\mathrm{P}<0.001)$ (Fig. 4J). These findings implied that NELL1, Runx2 and Msx2 may be involved in enhancing hPDLSC osteogenic differentiation, although it is unclear, our study is essential for understanding NELL1's potent osteoinduction effect. 
A
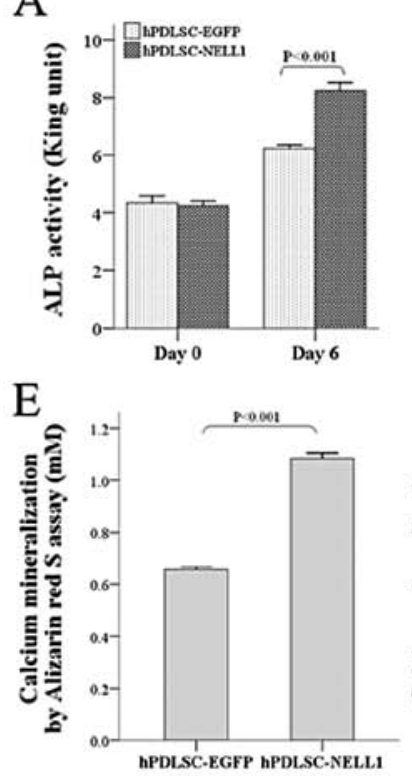

B
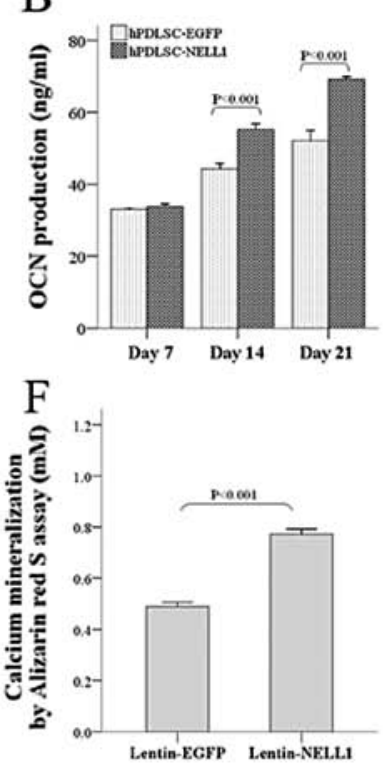
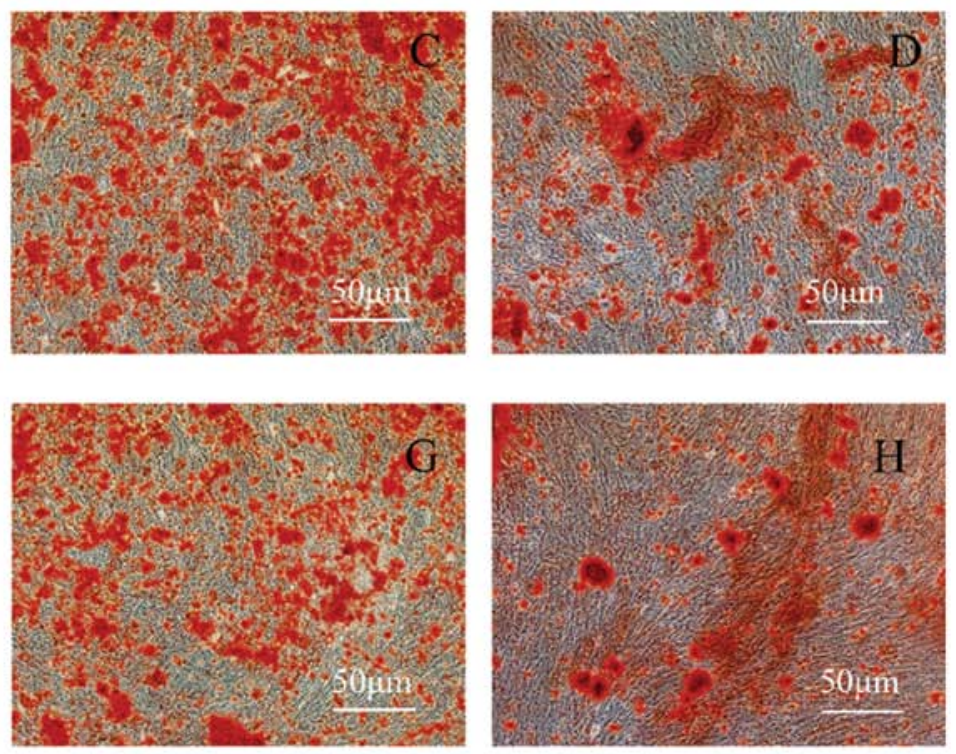
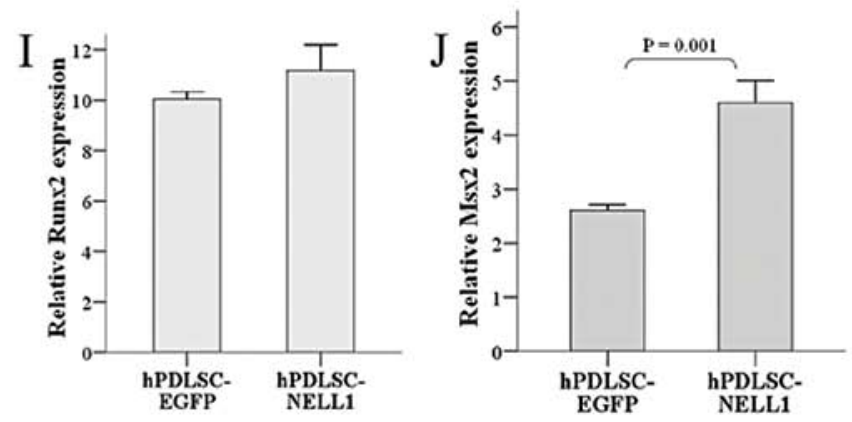

Figure 4. In vitro analysis of osteoblastic differentiation after hPDLSC transduction with Lenti-EGFP or Lenti-NELL1. (A) ALP activity of hPDLSCs transfected for 6 days. (B) OCN production in hPDLSCs transduced with Lenti-EGFP or Lenti-NELL1. (C-E) Transfected hPDLSCs were incubated in osteogenic media for 3 weeks. Calcium deposition was assessed by alizarin red staining. Quantitative analysis of calcium deposition of transfected cells. (F-H) Supernatant collected from Lenti-EGFP- or Lenti-NELL1-transfected cells was used to induce the osteogenic differentiation of hPDLSCs. Calcium deposition was quantitatively analyzed by alizarin red staining. (I and J) qPCR analysis of Runx2, and Msx2 in hPDLSCs transduced with Lenti-EGFP or Lenti-NELL1.

\section{Discussion}

PDLSC is a neural crest-deried stem cell with the charactistcs of neural ectoderm and mesoderm differentiation potential and potent plasticity $(22,25,26)$. Different from other MSCs, PDLSCs have enomous potential to form PDL, alveolar bone, and cementum and have been regarded as ideal seed cells in the treatment of craniofacial bone defects, especially for periodontal regeneration.

Unlike the gold-standard osteoinductive factors BMPs, NELL1 can only specifically act on osteochondral lineage and MSCs $(7,9,27)$, act as a crucial factor involved in the differentiation of neural crest cells into osteoblasts (28). For other nonosteochondral lineage, such as C2C12 myoblasts, NELL1 cannot independently induce osteogenesis (10). On the other hand, NELL1 does provide an advantage in the promotion of bone regeneration. Cowan et al (8) investigated the role of NELL1 in the suture of distracted palates of 4-week-old male rats. The results showed that NELL1 and BMP7 could significantly induce bone formation and newly formed bone in the NELL1 treated group was superior in mineralization and maturity of chondrocytes to that in the BMP7 group. To date, few studies have reported the effect of NELL1 on the osteoblast differentiation of hPDLSCs. In the present study, our results confirmed that a NELL1-expressing lentivirus effectively transfected hPDLSCs, which expressed NELL1 over an extended time period. In addition, NELL1 could potently improve the osteogenesis of hPDLSCs in vitro.

Currently, it has been demonstrated that recombinant NELL1 protein can promote bone regeneration in bone-defect animal models $(8,9,11)$. When protein is applied, the dose is usually at a high level, which may induce potential side effects and increase therapeutic cost. Regional gene therapy may be a preferred stratege for delivering protein in specific anatomical sites. The selection of optimal vectors is crucial in regional gene therapy. Although studies have shown that adenovirus vectors encoding NELL1 can successfully promote bone regeneration $(7,15)$, the target gene introduced by an adenovirus is episomal with the risk of inducing host immune response. Furthermore, adenoviruses cannot express the target gene lasting adequate time $(16,17)$. Thus, when repair large bone defects with adenovirus vectors, sufficient amounts and sustained delivery of NELL1 cannot be assured. Lentiviruses can integrate DNA into the host genome, resulting in long-term expression of the target protein $(17,29)$. Previous studies have shown that NELL1 expression in AdNELL1-transfected MC3T3 cells reached a peak level 3 days 
after transfection, but this rapidly decreased at 6 days after transfection (9). In the present study, Lenti-NELL1-transfected cells presented green fluorescence under a fluorescence microscope during the entire study period (6 weeks) (data not shown). Furthermore, qPCR also verified that at 14 days after transfection, NELL1 mRNA expression was still at a high level. Safety must be considered seriously before viral vectors are applied. In our study, the high efficient packaging system of the third lentivirus was used, which was less likely to produce replicationcompetent virus (RCV) $(30,31)$. Next, we will further validate the efficacy of Lenti-NELL1 modified hPDLSCs compared with those obtained by adenovirus for periodontal tissue regeneration in rodent models.

Although our results demonstrated that NELL1 could effectively enhance the osteogenic differentiation of hPDLSCs, the potential mechanism of this process was still unclear. Thus, qPCR was further applied to measure the mRNA expression of Runx2 and Msx2 in PDLSCs overexpressing NELL1 after osteogenic induction. Runx 2 is a key factor related to osteogenesis, as it can control the osteogenic differentiation of stromal cells through temporarily activating or inhibiting the growth and gene expression of stromal cells (32). The results showed that, although NELL1 overexpression could improve the osteogenic differentiation of hPDLSCs, cells overexpressing NELL1 had comparable Runx 2 expression to those in the control group. This may be attributed to the hierarchical relationship between NELL1 and Runx2 in the signaling pathway. Studies have found that NELL1 might be a downstream target of Runx2. Runx 2 can upregulate NELL1 expression by binding to the osteoblast-specific cis-acting element 2 (OSE2) in its promoter $(23,33)$. Our study found that overexpssion of NELL1 in hPDLSCs had no effect to Runx 2 expression comparing to the control group consistent with other findings (23). It implies that Runx 2 may exert its osteogenic effect via regulation of NELL1 expression. Insterstingly, Msx 2 has similar functions to NELL1. Studies have revealed that Msx 2 mutation is related to Boston type CS (24). Currently, roles of Msx2 in osteogenesis is controversial. Genetic analysis in human diseases and animal models have shown Msx2's positive role in improving osteogenesis $(24,34)$; while in vitro study has revealed that Msx2 can inhibit osteogenesis (35). There is evidence that NELL1 overexpression-induced craniofacial abnormalities in animal models are similar to those in animals with Msx2 overexpression (28). In both animal models, cranial suture overgrowth and increased incidence of exencephaly were noted. Currently, roles of NELL1 and Msx2 in craniofacial bone development, CS, and osteogenic differentiation are still unclear. The results in the present study showed that NELL1 overexpression could significantly upregulate $M s x 2$ expression. A prior study showed that the NELL1 promoter contains an Msx 2 binding sequence, NELL1 expression is regulated by Msx2, and Msx2-transfected fetal rat calvarial cells have a reduced expression of NELL1 (34). Combined with our findings, it suggested that NELL1 and Msx2 may intricately interrelate during osteogenesis, and more studies are required to elucidate their mechanism of enhancing osteogenisis.

In summary, the above findings suggested Lenti-NELLI transfection of hPDLSCs leads to overexpression of NELL1, which further improved osteogenesis of these cells in vitro. However, in vivo study was not performed to validate the above findings. Our group is conducting the application of NELL1-transfected PDLSCs to repair alveolar bone defects in animal models. We expect that the findings in this study will consummate the investigation about NELL1's osteoinductive effect on PDLSCs and provide a basis for further studies on the application of NELL1 as a growth factor and PDLSCs as seed cells in bone regeneration and periodontal tissue regeneration.

\section{Acknowledgements}

This study was supported by the National Natural Science Foundation of China. We thank associate Professor H. Wang at the Institute of Radiation of Academy of Military Medical Sciences and the associate Professor S.Y. Si at the Central Laboratory of the 306th Hospital of PLA for their kind help.

\section{References}

1. Elter JR, Offenbacher S, Toole JF and Beck JD: Relationship of periodontal disease and edentulism to stroke/TIA. J Dent Res 82: 998-1001, 2003.

2. Ducy P and Karsenty G: The family of bone morphogenetic proteins. Kidney Int 57: 2207-2214, 2000.

3. Rivera-Feliciano J and Tabin CJ: Bmp2 instructs cardiac progenitors to form the heart-valve-inducing field. Dev Biol 295: 580-588, 2006.

4. Franz M, Berndt A, Wehrhan F, Schleier P, Clement J and Hyckel P: Ectopic bone formation as a complication of surgical rehabilitation in patients with Moebius' syndrome. J Craniomaxillofac Surg 35: 252-257, 2007.

5. Mroz TE, Wang JC, Hashimoto R and Norvell DC: Complications related to osteobiologics use in spine surgery: a systematic review. Spine (Phila Pa 1976) 35 (Suppl 9): S86-S104, 2010.

6. Ting K, Vastardis H, Mulliken JB, et al: Human NELL-1 expressed in unilateral coronal synostosis. J Bone Miner Res 14: 80-89, 1999.

7. Aghaloo T, Jiang X, Soo C, et al: A study of the role of nell-1 gene modified goat bone marrow stromal cells in promoting new bone formation. Mol Ther 15: 1872-1880, 2007.

8. Cowan CM, Cheng S, Ting K, et al: Nell-1 induced bone formation within the distracted intermaxillary suture. Bone 38: 48-58, 2006.

9. Aghaloo T, Cowan CM, Chou YF, et al: Nell-1-induced bone regeneration in calvarial defects. Am J Pathol 169: 903-915, 2006.

10. Cowan CM, Jiang X, Hsu T, et al: Synergistic effects of Nell-1 and BMP-2 on the osteogenic differentiation of myoblasts. J Bone Miner Res 22: 918-930, 2007.

11. Siu RK, Lu SS, Li W, et al: Nell-1 protein promotes bone formation in a sheep spinal fusion model. Tissue Eng Part A 17: 1123-1135, 2011

12. Louis-Ugbo J, Kim HS, Boden SD, et al: Retention of 125Ilabeled recombinant human bone morphogenetic protein-2 by biphasic calcium phosphate or a composite sponge in a rabbit posterolateral spine arthrodesis model. J Orthop Res 20: 10501059, 2002.

13. Kofron MD and Laurencin CT: Orthopaedic applications of gene therapy. Curr Gene Ther 5: 37-61, 2005.

14. Baltzer AW and Lieberman JR: Regional gene therapy to enhance bone repair. Gene Ther 11: 344-350, 2004.

15. Lu SS, Zhang X, Soo C, et al: The osteoinductive properties of Nell-1 in a rat spinal fusion model. Spine J 7: 50-60, 2007.

16. Feeley BT, Conduah AH, Sugiyama O, Krenek L, Chen IS and Lieberman JR: In vivo molecular imaging of adenoviral versus lentiviral gene therapy in two bone formation models. J Orthop Res 24: 1709-1721, 2006.

17. Virk MS, Conduah A, Park SH, et al: Influence of short-term adenoviral vector and prolonged lentiviral vector mediated bone morphogenetic protein-2 expression on the quality of bone repair in a rat femoral defect model. Bone 42: 921-931, 2008.

18. Seo BM, Miura M, Gronthos S, et al: Investigation of multipotent postnatal stem cells from human periodontal ligament. Lancet 364: 149-155, 2004. 
19. Tu Q, Valverde $\mathrm{P}$ and Chen J: Osterix enhances proliferation and osteogenic potential of bone marrow stromal cells. Biochem Biophys Res Commun 341: 1257-1265, 2006.

20. James AW, Pan A, Chiang M, et al: A new function of Nell-1 protein in repressing adipogenic differentiation. Biochem Biophys Res Commun 411: 126-131, 2011.

21. Lee SJ, Kang SW, Do HJ, et al: Enhancement of bone regeneration by gene delivery of BMP2/Runx2 bicistronic vector into adipose-derived stromal cells. Biomaterials 31: 5652-5659, 2010.

22. Techawattanawisal W, Nakahama K, Komaki M, Abe M, Takagi Y and Morita I: Isolation of multipotent stem cells from adult rat periodontal ligament by neurosphere-forming culture system. Biochem Biophys Res Commun 357: 917-923, 2007.

23. Truong T, Zhang X, Pathmanathan D, Soo C and Ting K: Craniosynostosis-associated gene nell-1 is regulated by runx 2 . J Bone Miner Res 22: 7-18, 2007.

24. Wilkie AO, Tang Z, Elanko N, et al: Functional haploinsufficiency of the human homeobox gene MSX2 causes defects in skull ossification. Nat Genet 24: 387-390, 2000.

25. Widera D, Grimm WD, Moebius JM, et al: Highly efficient neural differentiation of human somatic stem cells, isolated by minimally invasive periodontal surgery. Stem Cells Dev 16 : 447-460, 2007.

26. Coura GS, Garcez RC, de Aguiar CB, Alvarez-Silva M, Magini RS and Trentin AG: Human periodontal ligament: a niche of neural crest stem cells. J Periodontal Res 43: 531-536, 2008.

27. Zhang X, Carpenter D, Bokui N, et al: Overexpression of Nell-1, a craniosynostosis-associated gene, induces apoptosis in osteoblasts during craniofacial development. J Bone Miner Res 18 2126-2134, 2003.
28. Zhang X, Kuroda S, Carpenter D, et al: Craniosynostosis in transgenic mice overexpressing Nell-1. J Clin Invest 110: 861-870, 2002 .

29. Miyazaki M, Sugiyama O, Zou J, et al: Comparison of lentiviral and adenoviral gene therapy for spinal fusion in rats. Spine (Phila Pa 1976) 33: 1410-1417, 2008.

30. Naldini L, Blomer U, Gallay P, et al: In vivo gene delivery and stable transduction of nondividing cells by a lentiviral vector. Science 272: 263-267, 1996.

31. Kafri T, Blomer U, Peterson DA, Gage FH and Verma IM: Sustained expression of genes delivered directly into liver and muscle by lentiviral vectors. Nat Genet 17: 314-317, 1997.

32. Yoshida CA, Furuichi T, Fujita T, et al: Core-binding factor beta interacts with Runx 2 and is required for skeletal development. Nat Genet 32: 633-638, 2002.

33. Zhang X, Ting K, Bessette CM, et al: Nell-1, a key functional mediator of Runx2, partially rescues calvarial defects in Runx2(+/-) mice. J Bone Miner Res 26: 777-791, 2011.

34. Zhou YX, Xu X, Chen L, Li C, Brodie SG and Deng CX: A Pro250Arg substitution in mouse Fgfrl causes increased expression of Cbfa1 and premature fusion of calvarial sutures. Hum Mol Genet 9: 2001-2008, 2000.

35. Shirakabe K, Terasawa K, Miyama K, Shibuya H and Nishida E: Regulation of the activity of the transcription factor Runx 2 by two homeobox proteins, Msx 2 and Dlx5. Genes Cells 6: $851-856$, 2001. 